\title{
Hybrid Energy Cells for Simultaneously Harvesting Multi-types of Energies
}

\author{
Ya Yang, ${ }^{\dagger * *}$ and Zhong Lin Wang ${ }^{\not,+}, *$
}

${ }^{\dagger}$ Beijing Institute of Nanoenergy and Nanosystems, Chinese Academy of Sciences, China

${ }^{t}$ School of Materials Science and Engineering, Georgia Institute of Technology, Atlanta, Georgia 30332-0245, United States

*To whom correspondence should be addressed: Email: yayang@binn.cas.cn; zlwang@gatech.edu

\begin{abstract}
ABSTRCT: Harvesting multi-mode energies from our living environment is an effective approach for solving the power source issue of the sensors and some personal electronics. Usually, the mechanical, thermal, and solar energies can be extensively found in our living environment. However, these energies are not always available at the same time, which is depending on the weather, working conditions, and some other cases. The concept of a hybrid energy cell is to develop a technology to individually or simultaneously scavenge multi-mode energies from environment, so that the sensors or other devices can sustainably work without the external power sources. In this paper, we review the investigations about the hybrid energy cells that include the energy harvesting units such as the piezoelectric nanogenerator, triboelectric nanogenerator, pyroelectric nanogenerator, thermoelectric generator and solar cells. The fabricated hybrid energy cells have been utilized to light up some electronics and for some self-powered electro-chemical applications. The obtained energies can be also stored in Li-ion battery, where the hybrid structure between the nanogenerator and the Li-ion battery exhibits the better charging performance than that of the conventional charging method. These investigations are of critical importance for sensing, medical science, environmental monitoring, defense technology, and even personal electronics.
\end{abstract}

KEYWORDS: hybrid energy cells, piezoelectric nanogenerator, triboelectric nanogenerator, pyroelectric nanogenerator, Li-ion battery 


\section{Introduction}

Our living environment has an abundance of energies in the forms of mechanical, optical, thermal and chemical energies. Scavenging these types of energies is of critical importance for long-term energy needs and sustainable development [1]. However, these energies are not always available at the same time, which depends on the weather, working conditions, and some other cases. The purpose for developing the hybrid energy cells is to simultaneously/individually scavenge these energies by using an integrated device, so that the devices/sensors can be continuously powered by using whatever energy that is available at their environment. The hybrid energy cells have the potential for fully utilizing the multimode energies in the environment under which the sensors/devices will be continuously and stably operating.

In last 5 years, we have developed many kinds of hybrid energy cells for simultaneously harvesting multi-types of energies, which not only can enhance the conversion efficiency of energy devices, but also has the potential applications in the self-powered electronics and some electrochemical reactions. The different energy harvesting units can work simultaneously or individually, and can be integrated in serial and parallel for enhancing the output voltage and current, respectively. The object of this paper is to give a summary about the development of the hybrid energy cells. These investigations have demonstrated many innovative approaches for developing integrated technologies for effectively harvesting available energies in our environment.

\section{Hybridization based on piezoelectric nanogenerators}

\subsection{Hybrid piezoelectric nanogenerator and solar cell for harvesting mechanical and solar energies}

In 2009, a hybrid energy cell was intended for simultaneously scavenging the mechanical and solar energies [2]. As shown in Figure 1a, the hybrid energy cell consists of a dye-sensitized solar cell on the top surface for scavenging the solar energy and a piezoelectric nanogenerator on the bottom surface for harvesting ultrasonic wave energy from the surroundings. Figure $1 \mathrm{~b}$ and 1c illustrate the SEM images of the $\mathrm{ZnO}$ nanowires, which were used as functional materials in both the solar cell and the piezoelectric nanogenerator. In the solar cell, $\mathrm{ZnO}$ nanowires coated with dye molecules were separated from the counter electrode by a gap in the electrolyte. In the piezoelectric nanogenerator, the $\mathrm{ZnO}$ nanowires were periodically bent/deflected by the ultrasonic wave to approach and contact the top zigzag electrode to realize the current/voltage output. Figure 1d displays the working principle of the hybrid energy cell by using the electron energy band diagram. The working of piezoelectric nanogenerator is due to the strained piezoelectric $\mathrm{ZnO}$ nanowire to drive the electrons to move from $\mathrm{ZnO}$ into Pt electrode under the forward biased Schottky barrier. Moreover, when a light was applied on the glass side of the solar cell, 
the electrons can be excited to a high energy state of the dye molecules and transferred to the conduction band of $\mathrm{ZnO}$, and then exported through the cathode. When the ultrasonic wave was off and the sun light source was on, the solar cell exhibited an open-circuit voltage $\left(V_{o c}\right)$ of $0.591 \mathrm{~V}$ and a short-circuit current density $\left(J_{s c}\right)$ of $6.9 \mu \mathrm{A} / \mathrm{cm}^{2}$, as illustrated in Figure 1f (the blue curve). When both the sun light and ultrasonic wave were turned on, the $V_{o c}$ was enhanced to $0.6 \mathrm{~V}$, while the $J_{s c}$ remained at the 6.9 $\mu \mathrm{A} / \mathrm{cm}^{2}$ (the red curve in Figure $1 \mathrm{f}$ ). The increase of voltage $(9 \mathrm{mV})$ is just due to the output voltage of the piezoelectric nanogenerator.

To solve the solvent leakage and evaporation of the conventional dye-sensitized solar cell, an innovative approach was reported that convolutes an ultrasonic wave driven piezoelectric nanogenerator and a solid-state dye-sensitized solar cell into a single compact structure for concurrently scavenging mechanical and solar energies [3]. As depicted in Figure 2a, the main structure consists of two layers of $\mathrm{ZnO}$ nanowire arrays that were created in a "teeth to teeth" configuration. The solar cell was fabricated on the top of the device, which is finally coated with a layer of metal to serve as the one electrode for the piezoelectric nanogenerator. The bottom $\mathrm{ZnO}$ nanowire array acts as the piezoelectric structure for scavenging the mechanical energy. Figure $2 b$ shows the working principle of the hybrid energy cell by using the electron energy band diagram, clearly indicating the movement of electrons in the solar cell and the piezoelectric nanogenerator. Figure 2c illustrate a scanning electron microscopy image of the $\mathrm{ZnO}$ nanowires, where the length and the interspacing distance are about 2-3 $\mu \mathrm{m}$ and 400-700 nm, respectively. When the ultrasonic wave source was off and the full sunlight source was on, the device exhibited a $V_{o c}$ of $0.415 \mathrm{~V}$ and a $J_{s c}$ of $252 \mu \mathrm{A} / \mathrm{cm}^{2}$ (blue curve in Figure $2 \mathrm{~d}$ ). When both the sunlight and the ultrasonic wave were turned on, the $J_{s c}$ remained at $252 \mu \mathrm{A} / \mathrm{cm}^{2}$, while the $V_{o c}$ reached $0.433 \mathrm{~V}$, which is just due to the contribution of the piezoelectric nanogenerator.

\subsection{Hybrid piezoelectric and biochemical nanogenerator for harvesting mechanical and biochemical energies}

The hybrid energy cell consists of a piezoelectric poly(vinylidene fluoride) nanofiber nanogenerator and a flexible enzymatic bio-fuel cell for simultaneously scavenging the mechanical and biochemical energies [4]. As illustrated in Figure 3a, the piezoelectric nanogenerator was fabricated on a flexible substrate, so that the device can be deformed under alternating compressive and tensile force to produce a piezoelectric electric field for diving a flow of electrons back and forth through the external circuit. Figure $3 b$ displays the output voltages of the piezoelectric nanogenerator under the different strain rates, revealing that the output voltage can be increased with increasing the strain rates. As depicted in Figure $3 \mathrm{c}$, the output voltage of the biochemical cell unit increases with increasing the glucose concentration in PBS solution, where the voltage value is about several tens of micro voltages. The integration operation 
of the piezoelectric nanogenerator and the bio-fuel cell is presented in Figure 3d, clearly indicating that the two generator units can individually and simultaneously work for scavenging the mechanical and biochemical energies.

\subsection{Hybrid solar cell, piezoelectric and pyroelectric nanogenerators for harvesting solar, mechanical, and thermal energies}

To simultaneously scavenge the solar, mechanical and thermal energies, we fabricated a hybrid energy cell that consists of a $\mathrm{ZnO}$ nanowire array-poly(3-hexylthiophene) $\left(\mathrm{P}_{3} \mathrm{HT}\right)$ hetero-junction solar cell and the polarized poly(vinylidene fluoride) (PVDF) film-based piezoelectric and pyroelectric nanogenerators [5]. As illustrated in Figure 4a, the bottom piezoelectric/pyroelectric nanogenerator consists of two Ag electrodes and a layer of PVDF film. The top solar cell consists of an Ag electrode, $\mathrm{P}_{3} \mathrm{HT}$ film, and $\mathrm{ZnO}$ nanowire array on a transparent indium tin oxide (ITO) film electrode. Figure $4 \mathrm{~b}$ displays a scanning electron microscopy (SEM) image of the $\mathrm{ZnO}$ nanowire array, showing that the $\mathrm{ZnO}$ nanowires have the diameters of about $100 \mathrm{~nm}$. The cross-section SEM image of the $\mathrm{ZnO}$ nanowire array indicates that the length is about $10 \mu \mathrm{m}$, as displayed in the inset of Figure 4b. Figure 4c depicts the output voltage and current signals of the hybrid pyroelectric and piezoelectric nanogenerators. When there is a temperature change applied on the device, the pyroelectric nanogenerator can deliver an output voltage of about $3 \mathrm{~V}$ and an output current of about $30 \mathrm{nA}$. Moreover, when the periodic force was applied on the device, the piezoelectric nanogenerator can produce an output voltage of about $0.8 \mathrm{~V}$ and an output current of about $20 \mathrm{nA}$. When both the changing temperature and the periodic forces were applied on the device, the hybrid energy cell can deliver the two kinds of energies at the same time. Under AM 1.5 illumination with $100 \mathrm{~mW} / \mathrm{cm}^{2}$ light intensity, the open-circuit voltage and the short-circuit current of the solar cell are about $0.41 \mathrm{~V}$ and $31 \mu \mathrm{A} / \mathrm{cm}^{2}$ (Figure 4d), respectively. These results indicate that the fabricated hybrid energy cell can simultaneously and individually scavenge the mechanical, thermal, and solar energies.

\section{Hybridization based on triboelectric nanogenerator}

\subsection{Hybrid triboelectric and pyroelectric nanogenerators for harvesting mechanical and thermal energies}

In 2013, we fabricated a hybrid energy cell that consist of a triboelectric nanogenerator for harvesting the mechanical energy at the top and a pyroelectric nanogenerator for scavenging the thermal energy at the bottom [6]. As illustrated in Figure 5a, the triboelectric nanogenerator consists of a polyester (PET) film on the Ni electrode and a polydimethylsiloxane (PDMS) nanowire array on a ITO electrode, while the pyroelectric nanogenerator is based on a PZT film. Figure 5b illustrates a SEM image of the PDMS nanowire array, clearly showing that the PDMS nanowires have the diameters of about $200 \mathrm{~nm}$. As 
shown in Figure 5c, the hybrid energy cell can deliver an output current of about $0.4 \mu \mathrm{A}$ for the pyroelectric nanogenerator and an output current of about $0.3 \mu \mathrm{A}$ for the triboelectric nanogenerator. Since both the pyroelectric and the piezoelectric nanogenerators have the alternating electric (AC) output signals, the total output current was sometimes weakened (see the time from 400 to $550 \mathrm{~s}$ in Figure 5c), resulting in the decrease of the produced electricity. In order to solve this problem, all the output signals were rectified by the full-wave bridge circuits. As a result, all the output current signals are positive and the output current of the hybrid energy cell is always larger than that of individual nanogenerators, as displayed in Figure 5d.

\subsection{Hybrid triboelectric nanogenerator and solar cell for harvesting mechanical and solar energies}

When a solar cell is working in nature, wind becomes an existing source of energy together with the working environment of solar cell, where wind energy can be easily converted to electricity by using the flexible nanogenerators. An interesting question is what if the surface layer for protecting the solar cell can be utilized to scavenge the wind energy. We have demonstrated a hybrid energy cell that consists of a micropyramid Si solar cell and a flexible triboelectric nanogenerator serving as the protection layer for Si solar cell, for simultaneously scavenging the solar and mechanical energies [7]. As displayed in Figure 6a, the top triboelectric nanogenerator is based on the contact/separation between the transparent PDMS nanowire array and the ITO film. Figure $6 \mathrm{~b}$ presents a SEM image of the obtained PDMS nanowire array, revealing that the diameters of nanowires are about $200 \mathrm{~nm}$. The bottom solar cell is based on micropyramid $\mathrm{p}-\mathrm{n}$ junction with the layers of $\mathrm{Al} / \mathrm{p}^{+}$back surface field layer/p type Si layer/ $\mathrm{n}^{+}$ emitter layer/SiN film/Ag grids/ITO electrode. The inset in Figure 6b illustrates a SEM image of the Si pyramids, where the sizes of $\mathrm{Si}$ pyramids are from 1 to $10 \mu \mathrm{m}$. Figure 6c displays the output performances of the fabricated Si pyramid solar cell with and without the PDMS nanowire array on the device. Under the light illumination intensity of $100 \mathrm{~mW} / \mathrm{cm}^{2}$, the $V_{o c}$ and $I_{s c}$ of the solar cell without the PDMS nanowire array on it are about $0.6 \mathrm{~V}$ and $35 \mathrm{~mA} / \mathrm{cm}^{2}$, respectively. The conversion efficiency of the solar cell covered with the PDMS nanowire array can be decreased from 16\% to $14 \%$. Figure $6 \mathrm{~d}$ depicts the output voltage of the hybrid energy cell, where the output signals of the triboelectric nanogenerator have been rectified. It can be clearly seen that the hybrid energy cell can simultaneously and individually to scavenge the solar and mechanical energies, respectively. Moreover, Guo et al. fabricated a hybrid energy cell that can be utilized to simultaneously and individually harvest wind and light energy [8], where the triboelectric nanogenerator was used as the wind harvester.

\subsection{Hybrid solar cells, triboelectric and thermoelectric generators for harvesting solar, mechanical, and thermal energies}


As illustrated in Figure 7a, the hybrid energy cell consists of a thermoelectric generator at the bottom, a triboelectric nanogenerator at the middle of the device, and a Si-based solar cell at the top [9]. Figure $7 \mathrm{~b}$ displays that the triboelectric nanogenerator is based on the contact/separation between polyamide and perfluoroalkoxy films. Figure 7c shows the output performance of the triboelectric nanogenerator, where the corresponding output voltage and current are about $110 \mathrm{~V}$ and $60 \mu \mathrm{A}$, respectively. Figure $7 \mathrm{~d}$ depicts the output performance of the integrated thermoelectric generators and the solar cells in series. Under the light illumination, the output voltage and the output current of the hybrid energy cell are about $3.5 \mathrm{~V}$ and $30 \mathrm{~mA}$, respectively. Under both the light illumination and the heat applied at the bottom of the thermoelectric generator, the total output voltage and current of the two harvesters were about $5.2 \mathrm{~V}$ and $34 \mathrm{~mA}$, respectively. The fabricated hybrid energy cell can be utilized to effectively scavenge solar, mechanical, and thermal energies.

\section{Hybrid energy cells for self-powered personal electronics and electrochemical applications}

\subsection{Hybrid energy cell for lighting LCDs and charging a Li-ion battery}

The obtained energies by using the hybrid energy cells can be utilized to directly light up LCDs. As illustrated in Figure 8a, a LCD with a display of "666666" can be clearly observed, where the hybrid piezoelectric and pyroelectric nanogenerators were used to light up the LCD [5]. Moreover, it is necessary to store the produced energy in a Li-ion battery for the other uses. Figure $8 \mathrm{~b}$ displays the hybrid energy cell-charging and the subsequent constant-current discharging curves of a Li-ion battery [4]. The battery can be charged from 1.54 to $3.6 \mathrm{~V}$ in about $1.3 \mathrm{~h}$. Under the constant discharging current of about $10 \mathrm{~mA}$, the discharging of the battery can last for about $580 \mathrm{~s}$ before it returned back to $1.54 \mathrm{~V}$, resulting in the stored electric capacity is up to $1.61 \mathrm{mAh}$. The charged Li-ion battery can be utilized to power a red laser diode (the inset in Figure 8b) and tens of LEDs (Figure 8c) [10]. Figure 8d presents that a commercial cell phone can work after it was charged by using the hybrid energy cells.

\subsection{Hybrid energy cell for self-powered electro-degradation}

The feasibility of electrochemical degradation of dyes, such as methyl orange (MO), for wastewater treatment has been reported [11-13]. However, all these electrochemical degradations need external power sources, which largely limits the development of this technique. To solve this problem, we developed a hybrid energy cell to achieve self-powered electrochemical degradation of dyeing wastewater [6]. As shown in Figure 9a, the produced energy by the hybrid energy cell can be used to charge a Li-ion battery by using a rectification bridge circuit, and then was used for the electrodegradation of methyl orange. Figure $9 \mathrm{~b}$ illustrates that a Li-ion battery can be charged from 0.15 to $1 \mathrm{~V}$ in about $25000 \mathrm{~s}$ by using the fabricated hybrid energy cell. Before using the Li-ion battery for the 
electro-degradation, a control experimental was finished, as displayed in Figure 9c. Under this condition, there is no any change in the absorption spectra of the MO solution at the different time intervals. Figure $9 \mathrm{~d}$ presents that the UV-visible absorption spectra of the MO solution for electro-degradation by using a charged Li-ion battery. It can be clearly seen that the characteristic absorption peak (461 nm) intensity of $\mathrm{MO}$ decreases with increasing the electro-degradation time, indicating the decrease of the MO concentration. Without the $\mathrm{Li}$-ion battery as the storage unit, we also demonstrated that the pyroelectric nanogenerator can be directly utilized for electro-degradation of the MO solution, as illustrated in Figure 9e. The characteristic absorption peak intensity of $\mathrm{MO}$ decreases with increasing the degradation time, where the degradation percentage of MO is up to $80 \%$ after $144 \mathrm{~h}$. The optical images of MO solution before and after degradation clearly shows that the color of MO solution at $144 \mathrm{~h}$ was obviously shallowed as compared with that before the degradation, indicating that the MO has been effectively degraded by using the pyroelectric nanogenerator.

\subsection{Hybrid energy cell for self-powered water splitting}

Production of hydrogen by splitting water using the electrolysis effect is a potential source of clean and renewable energy. It has been discovered more than 2000 years ago that the electrolysis effect can be used to split the water into hydrogen [14]. However, an external power source for driving the oxidation or reduction reactions of $\mathrm{H}_{2} \mathrm{O}$ molecules is mandatory for electrolysis, which largely limits the development of this technology. To solve this problem, we can harvest energy from our living environment by using a hybrid energy cell that can be directly utilized for electrolysis without using an external power source [9]. As displayed in Figure 10a, there are two methods that can be utilized for the water splitting. When the point "1" was connected to the point "2", the produced energies can be stored in a Li-ion battery and then used for water splitting. When point " 1 " was connected to the point " 3 ", the produce energies can be directly used for splitting of water. Here, a Pt electrode was used as anode, while the cathode was inserted into a hydrogen collection tube, where the top end of the tube was sealed by using epoxy. Figure 10b illustrates an optical image of the whole system, where the hydrogen collection tube was fully filled with water solution, and it was then partly inserted into the water container. Due to the pressure of the atmosphere, the water solution cannot flow from the collected tube to $t$ he container. However, when the produced hydrogen was collected on the top of the tube, the water solution can flow out from the collection tube due to the larger pressure of the hydrogen, resulting in the drop of water surface level in the collected tube. Figure 10c depicts the optical images of hydrogen collection tube at the different times, where the hybrid energy cell was directly utilized to split the water. The obtained volume of the hydrogen increases with increasing the time, resulting in the drop of the water surface level in the collection tube, where a linear relationship between the volume of hydrogen 
and the time can be observed with a rate of $4 \times 10^{-4} \mathrm{~mL} / \mathrm{s}$, as displayed in Figure $10 \mathrm{~d}$.

\section{Self-charging power pack by hybridizing nanogenerator and energy storage unit}

Usually, the energy generation and storage are two distinct process that can be accomplished by using two separated units, such as the piezoelectric nanogenerator as the energy generation unit and the Li-ion battery as the energy storage unit. A self-charging power pack is based on the direct conversion of the mechanical energy into electricity in one process [15]. As illustrated in Figure 11a, the polyethylene separator for conventional Li-ion battery was replaced by a piezoelectric poly(vinylidene fluoride) (PVDF) film, resulting in that the piezoelectric potential from the PVDF film can act as a charge pump to drive Li ions to move from the cathode to the anode to realize the charging reactions at electrodes. Figure $11 \mathrm{~b}$ displays an optical image of a Li-ion battery based self-charging power pack under a shoe, suggesting that the mechanical energy from human motions can be directly converted into electricity. Figure 11c depicts a cross-sectional SEM image of the self-charging power cell, which is composed of nano-tubes as anode, piezoelectric polymer film as separator and cathode. The height and diameter of the nanotubes are about $20 \mu \mathrm{m}$ and $100 \mathrm{~nm}$, respectively, as shown in Figure 11d. Figure 11e illustrates a typical self-charging and discharging cycle. The voltage of the device can be increased from 327 to $395 \mathrm{mV}$ in about $240 \mathrm{~s}$ under the compressive force applied to the device at a frequency of $2.3 \mathrm{~Hz}$. Under a discharge current of $1 \mu \mathrm{A}$, the device can be discharged back to its original voltage of $327 \mathrm{mV}$ in about $130 \mathrm{~s}$, resulting in the stored electric capacity of about $0.036 \mu \mathrm{Ah}$. The fabricated self-powered charging power pack has the higher charging efficiency than the conventional generator-based charging process, as displayed in Figure 11f. By using the similar method, Xue et al. fabricated other selfcharging power cells to realize one-step energy conversion and storage $[\mathbf{1 6}, \mathbf{1 7}]$.

\section{Summary and perspectives}

As sparked by the first demonstration of the hybrid energy cells in 2009, research in hybrid energy cell-based energy conversion, storage and applications has inspired extensive interest. Hybrid energy cell is about the integration of the multi-mode energy harvesters to realize the sustainable powering of the sensors. The investigations of the hybrid energy cells require the high technology platform due to the knowledge basis of the muti-mode energy harvesters such as piezoelectric nanogenerator, triboelectric nanogenerator, pyroelectric nanogenerator, solar cells and so on. The research of hybrid energy cell will be focused on how to maximize the scavenged energies from the environment. We anticipate that much more enhancement of the total output power density will be demonstrated in the next few years. This huge enhancement of the output performance can make it possible for solving the power source issue of some personal electronics such as the cell phones or some other smart products. 
The hybrid energy cell-based self-powered energy pack will be a new paradigm for realizing the sustainable working of the micro/nanosystems.

\section{ACKNOWLEDGMENTS}

Research was supported by BES DOE, NSF, and the Knowledge Innovation Program of the Chinese Academy of Sciences (KJCX2-YW-M13), NSFC (NO. 61404034) and the "thousands talents" program for pioneer researcher and his innovation team, China. We thank Chen $\mathrm{Xu}$, Hulin Zhang, Xinyu Xue, Ying Liu, Sihong Wang, Long Lin, Sangmin Lee, and Yan Liu for their contributions to the works reviewed here.

\section{REFERENCES}

[1] M. S. Dresselhaus, I. L. Thomas, Nature 414 (2001) 332-337.

[2] C. Xu, X. Wang, Z. L. Wang, J. Am. Chem. Soc. 131 (2009) 5866-5872.

[3] C. Xu, Z. L. Wang, Adv. Mater. 23 (2011) 873-877.

[4] B. J. Hansen, Y. Liu, R. Yang, Z. L. Wang, ACS Nano 4 (2010) 3647-3652.

[5] Y. Yang, H. Zhang, G. Zhu, S. Lee, Z.-H. Lin, Z. L. Wang, ACS Nano 7 (2013) 785-790.

[6] Y. Yang, H. Zhang, S. Lee, D. Kim, W. Hwang, Z. L. Wang, Nano Lett. 13 (2013) 803-808.

[7] Y. Yang, H. Zhang, Y. Liu, Z.-H. Lin, S. Lee, Z. Lin, C. P. Wong, Z. L. Wang, ACS Nano 7 (2013) 2808-2813.

[8] H. Guo, X. He, J. Zhong, Q. Zhong, Q. Leng, C. Hu, J. Chen, L. Tian, Y. Xi, J. Zhou, J. Mater. Chem. A 2 (2014) 2079-2087.

[9] Y. Yang, H. Zhang, Z.-H. Lin, Y. Liu, J. Chen, Z. Lin, Y. S. Zhou, C. P. Wong, Z. L. Wang, Energy Environ. Sci. 6 (2013) 2429-2434.

[10] Y. Yang, H. Zhang, J. Chen, S. Lee, T.-C. Hou, Z. L. Wang, Energy Environ. Sci. 6 (2013) 17441749.

[11] M. Panizza, G. Cerisola, Environ. Sci. Technol. 38 (2004) 5470-5475.

[12] N. Mohan, N. Balasubramanian, C. A. Basha, J. Hazard. Mater. 147 (2007) 644-651.

[13] C. D. Vecitis, G. Gao, H. Liu, J. Phys. Chem. C. 115 (2011) 3621-3629. 
[14] K. Sun, Y. Jing, C. Li, X. Zhang, R. Aguinaldo, A. Kargar, K. Madsen, K. Banu, Y. Zhou, Y.

Bando, Z. Liu, D. Wang, Nanoscale 4 (2012) 1515-1521.

[15] X. Y. Xue, S. H. Wang, W. X. Guo, Y. Zhang, Z. L. Wang, Nano Lett. 12 (2012) 5048-5054.

[16] X. Y. Xue, P. Deng, B. He, Y. Nie, L. Xing, Y. Zhang, Z. L. Wang, Adv. Energy Mater. 4 (2014) 1301329.

[17] L. Xing, Y. Nie, X. Xue, Y. Zhang, Nano Energy 10 (2014) 44-52.

\section{FIGURE CAPTIONS}

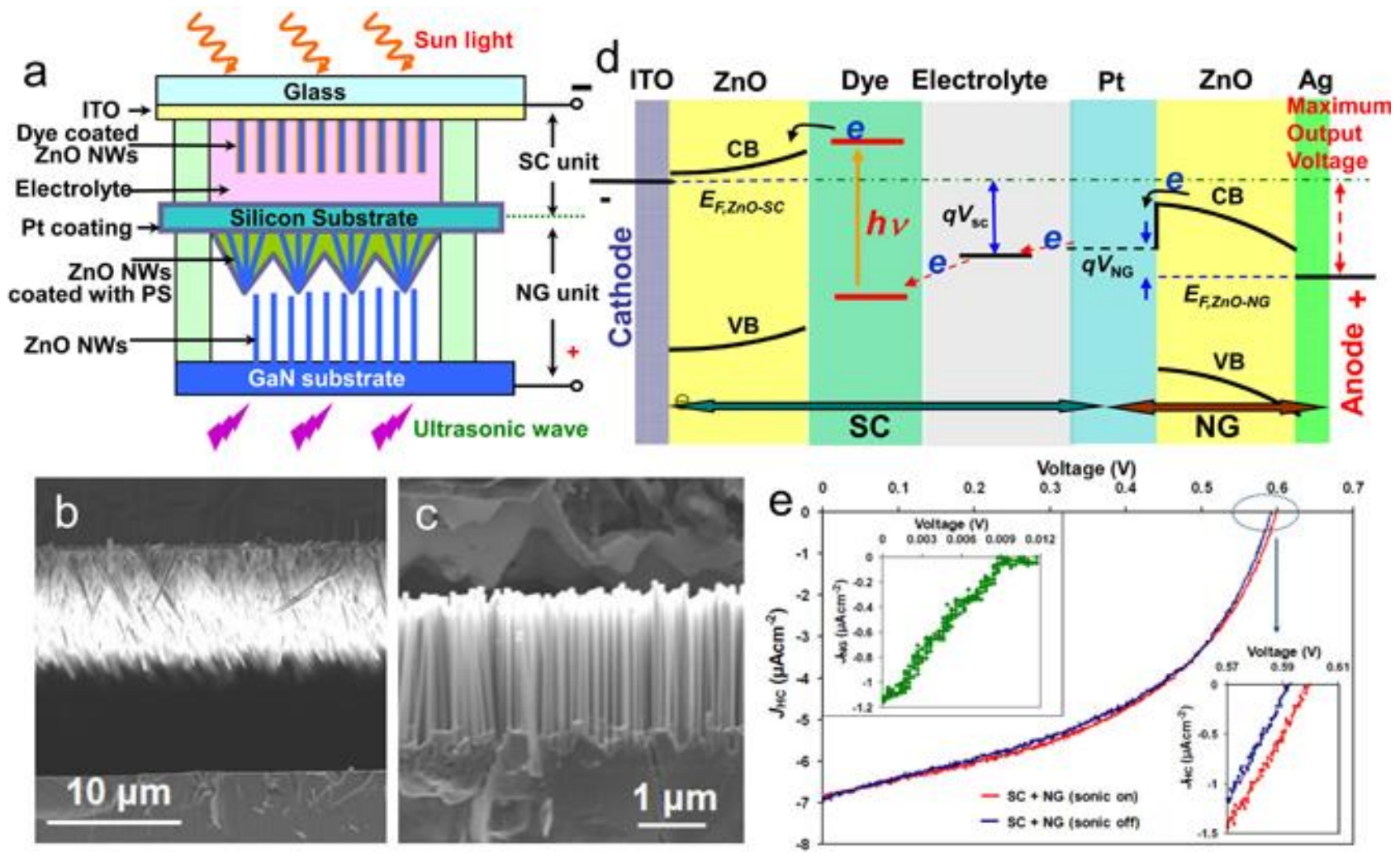

Figure 1. (a) Schematic diagram of serially integrated hybrid energy cell. (b) SEM image of the solar cell unit. (c) SEM image of the nanogenerator unit. (d) Electron energy band diagram of the hybrid energy cell. (e) A comparison of $J-V$ characteristics of a hybrid energy cell when illuminated by simulated sun light with (red curve) and without (blue curve) turning on the ultrasonic wave excitation. The inset is an expanded output of the open-circuit voltage points around the axial cross point. Reproduced form ref 2. Copyright 2009 American Chemical Society. 

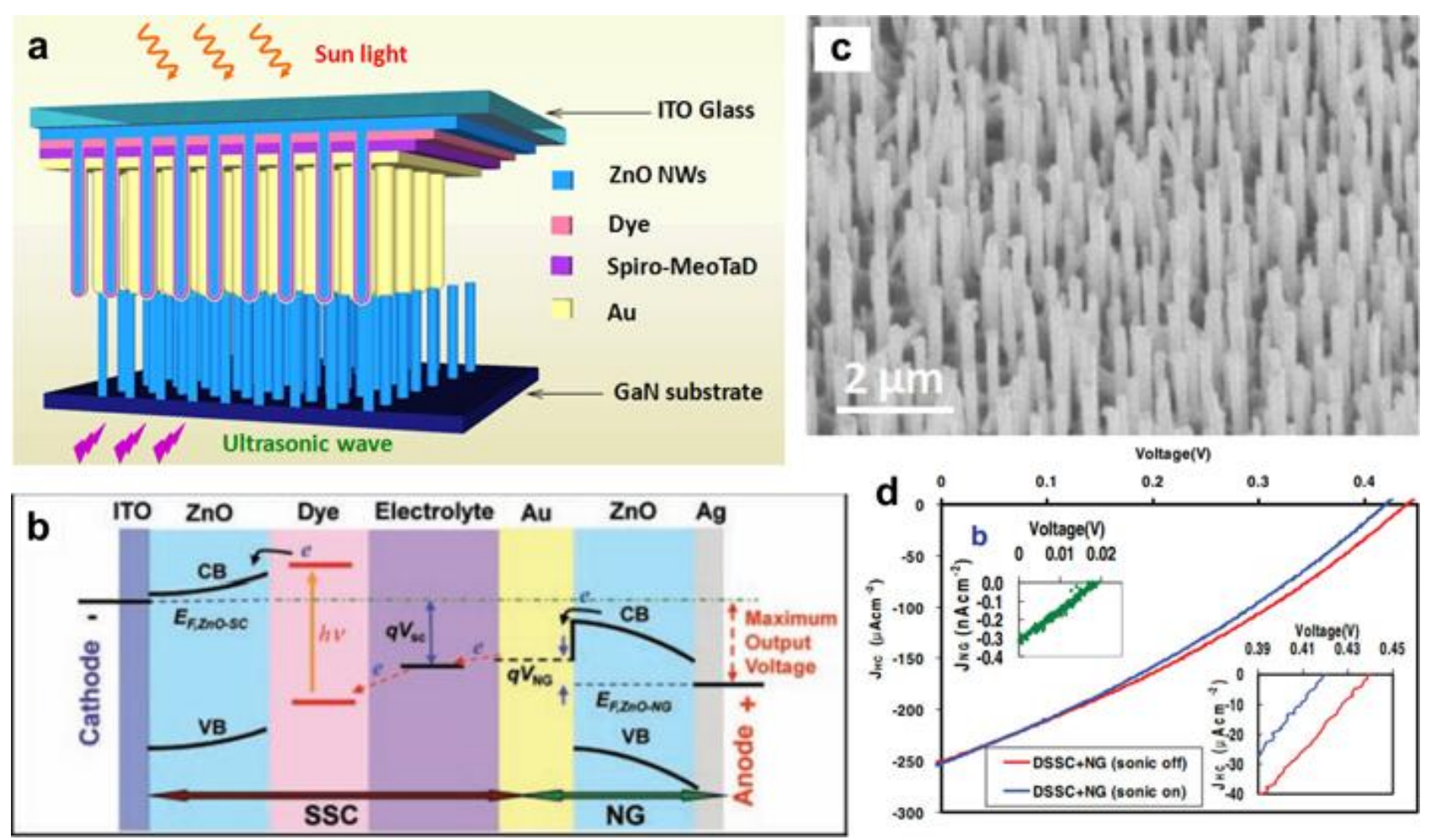

Figure 2. (a) Schematic structure of a hybrid energy cell. (b) Electron energy band diagram of the hybrid energy cell. (c) SEM image of the as-grown $\mathrm{ZnO}$ nanowire array using the high-temperature vapor deposition method for fabricating the nanogenerator. (d) A comparison in power output J-V characteristics of a hybrid energy cell. Reproduced form ref 3. Copyright 2011 Wiley. 

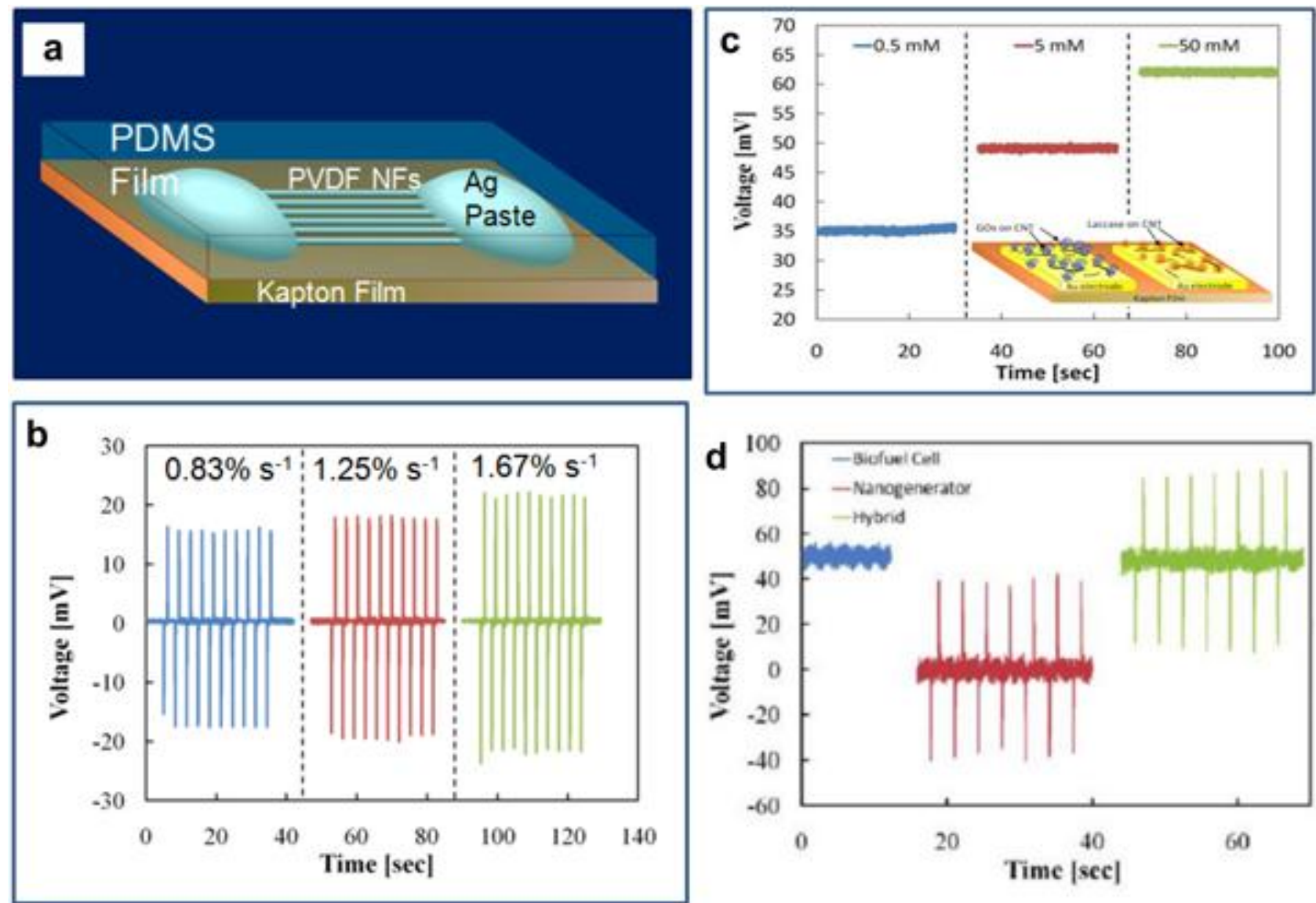

Figure 3. (a) The PVDF nanofiber lies on a Kapton substrate, with both ends bonded with silver paste and the entire device encapsulated with PDMS. (b) Output voltages of the PVDF nanogenerator as a function of strain rate with a maximum strain of about $0.05 \%$. (c) Output voltages of the biofuel cell as a function of glucose concentration in PBS solution. The inset image shows a schematic illustration of the fabricated device. (d) Output voltages of the independent and combined operation of the two nanogenerators. Reproduced form ref 4. Copyright 2010 American Chemical Society. 

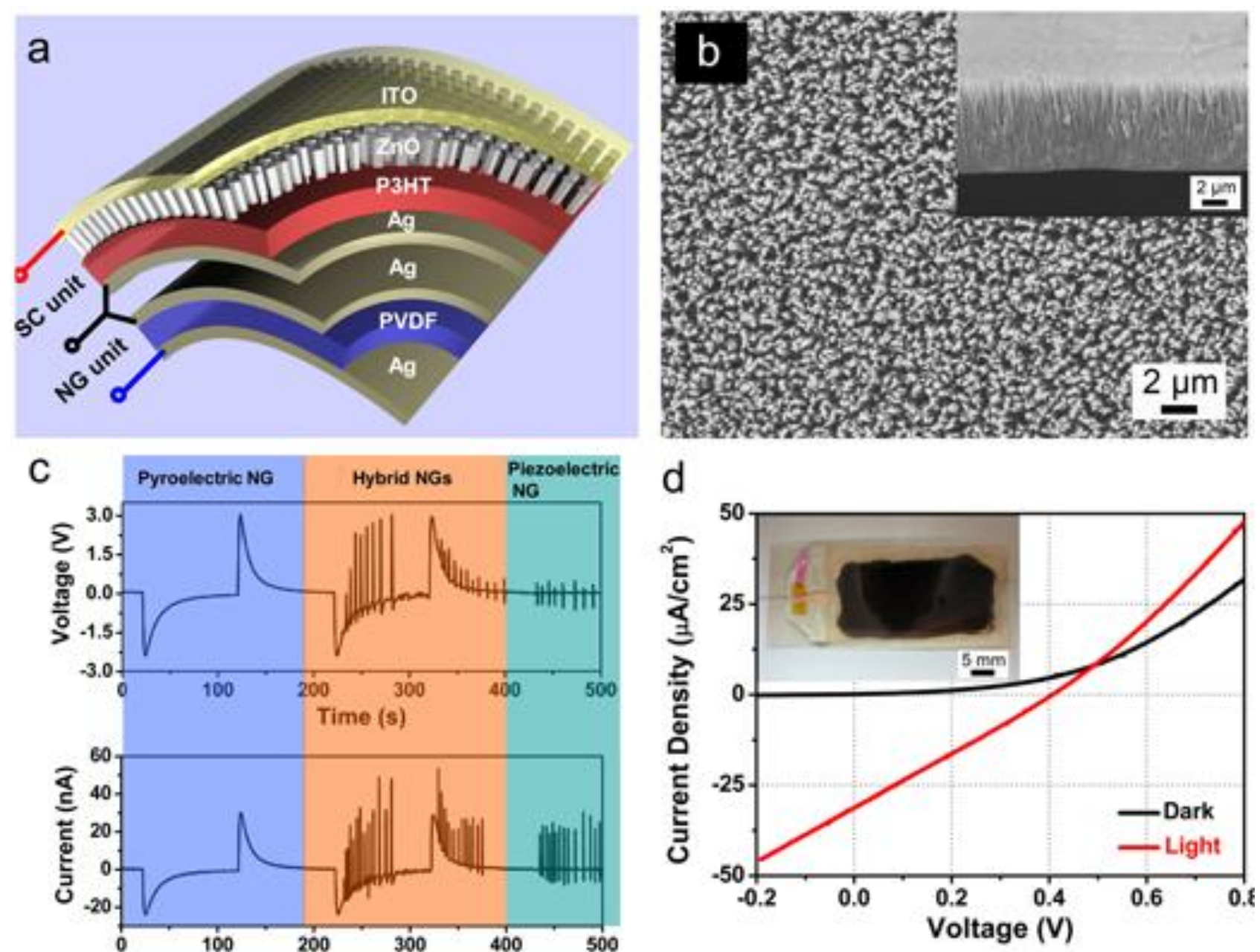

Figure 4. (a) Schematic diagram of the fabricated hybrid energy cell. (b) SEM image of the $\mathrm{ZnO}$ nanowire array. (c) Output voltage and current of the hybrid pyroelectric and piezoelectric nanogenerators. (d) $I-V$ characteristics of the device under dark and light illumination conditions. The inset shows a photograph of the $\mathrm{ZnO}$ nanowire array- $\mathrm{P}_{3} \mathrm{HT}$ film solar cell. Reproduced form ref 5 . Copyright 2013 American Chemical Society. 

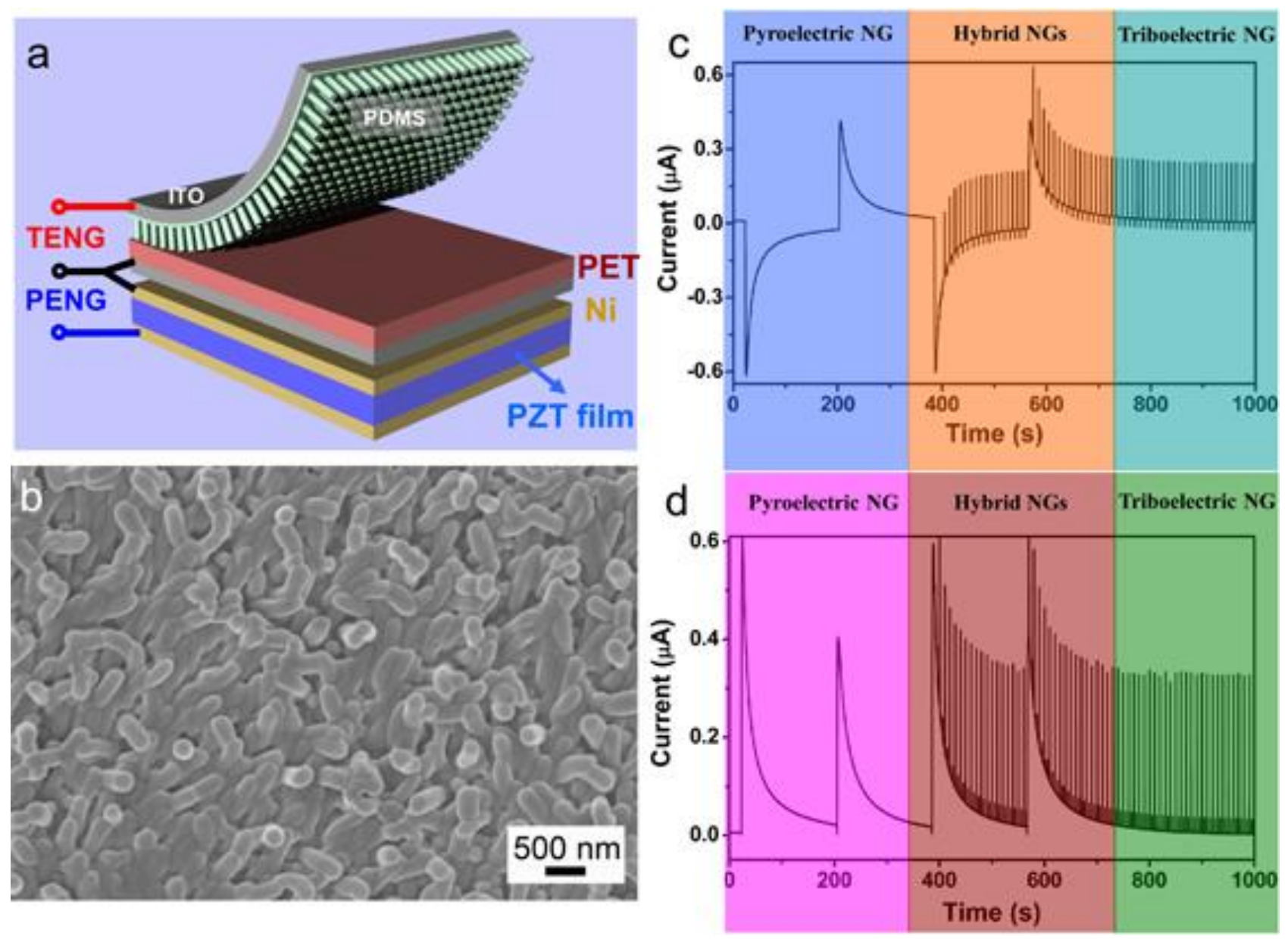

Figure 5. (a) Schematic diagram of the fabricated hybrid energy cell. (b) Enlarged SEM image of the PDMS nanowire array. (c) Output current of the hybrid energy cell, where the triboelectric and pyroelectric nanogenerators can work simultaneously and individually. (d) Output current of the hybrid energy cell after the output signals were rectified by a full-wave bridge circuit. Reproduced form ref 6. Copyright 2013 American Chemical Society. 

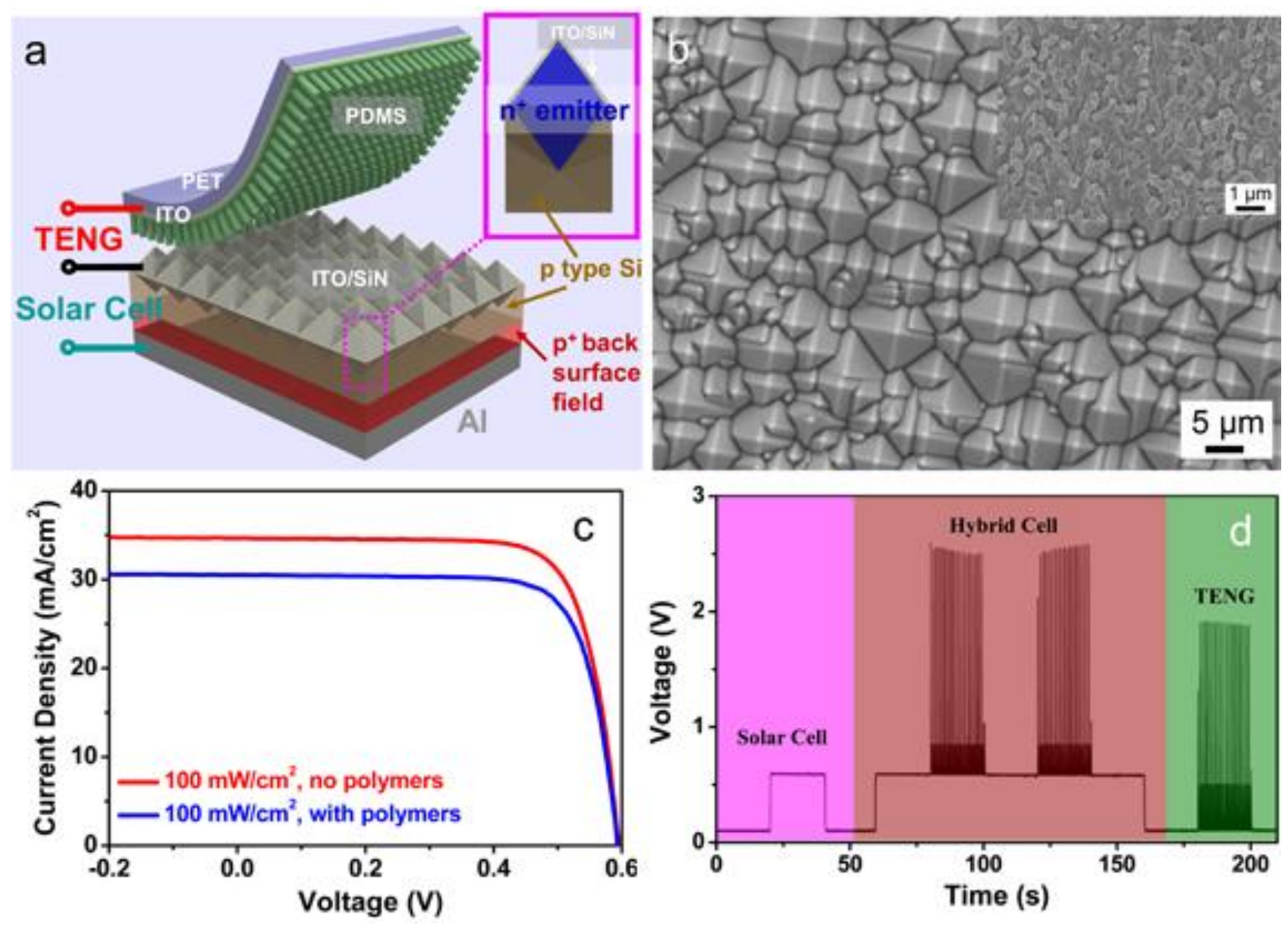

Figure 6. (a) Schematic diagram of the fabricated hybrid energy cell. (b) SEM image of the fabricated Si pyramids. The inset shows a SEM image of the PDMS nanowire array. (c) $J-V$ curves of the Si solar cell covered with and without PET-PDMS polymers under the light illumination intensity of 100 $\mathrm{mW} / \mathrm{cm}^{2}$. (d) Output voltage of the hybrid solar cell and triboelectric nanogenerator for scavenging solar and mechanical energies. Reproduced form ref 7. Copyright 2013 American Chemical Society. 

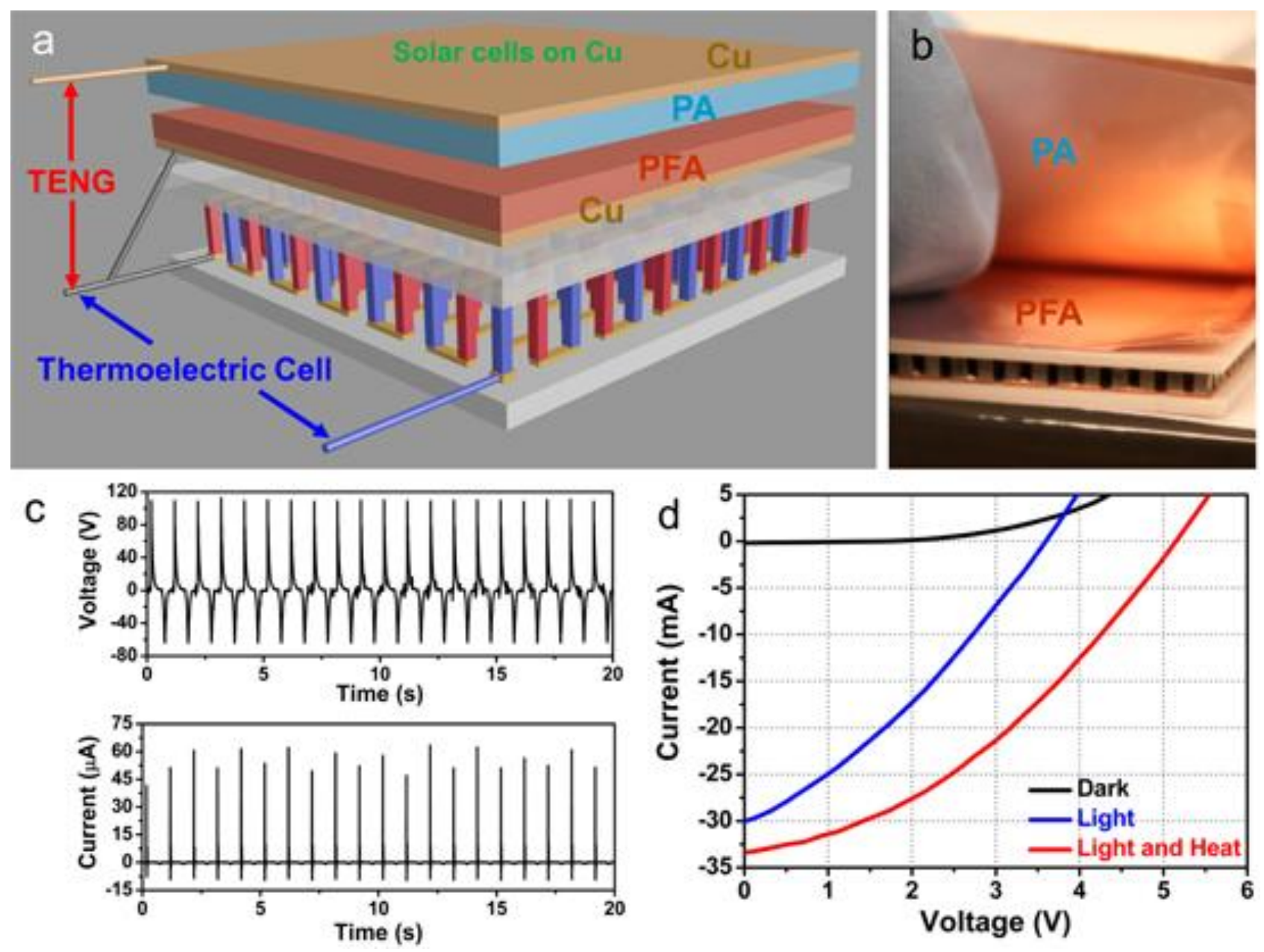

Figure 7. (a) Schematic diagram of the fabricated hybrid energy cell including a triboelectric nanogenerator, a thermoelectric cell, and solar cells at the top. (b) Photograph of the hybrid energy cell. (c) Output voltage and current of the triboelectric nanogenerator. (d) Current-voltage characteristics of the hybrid energy cell under the different conditions. Reproduced form ref 9. Copyright 2013 Royal Society of Chemistry. 

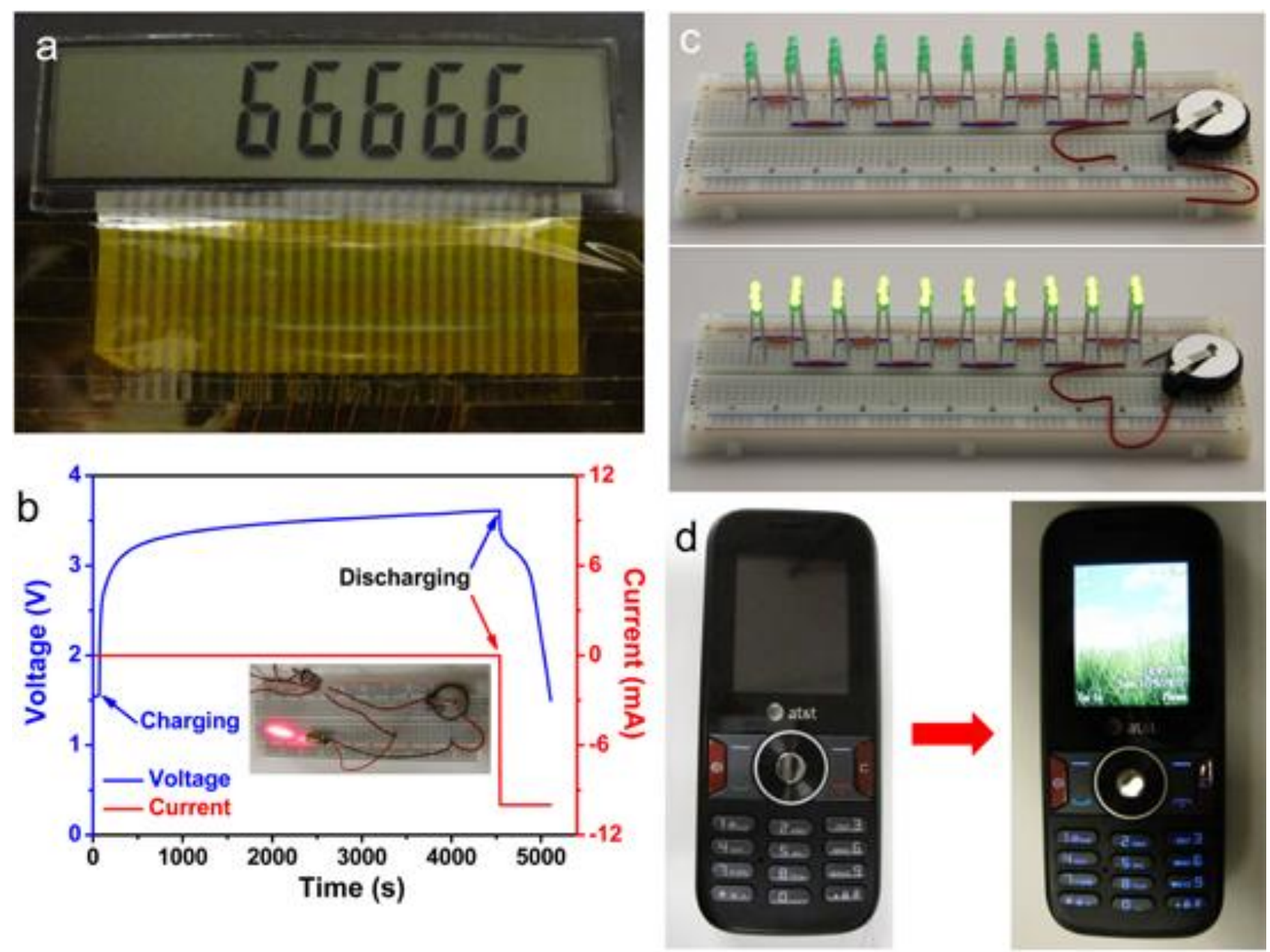

Figure 8. (a) Photograph of the lighted LCD by the hybrid energy cell. (b) The hybrid energy cellcharging and the subsequent constant-current discharging curves of a Li-ion battery. The inset shows an optical image, where a red laser diode was driven by the charged Li-ion battery. (c) Photograph of the lighted LEDs that were driven by the charged Li-ion battery. (d) Photograph of a charged cell phone by using the hybrid energy cell. Reproduced form refs 5, 7 and 10. Copyright 2013 American Chemical Society and Copyright 2013 Royal Society of Chemistry. 

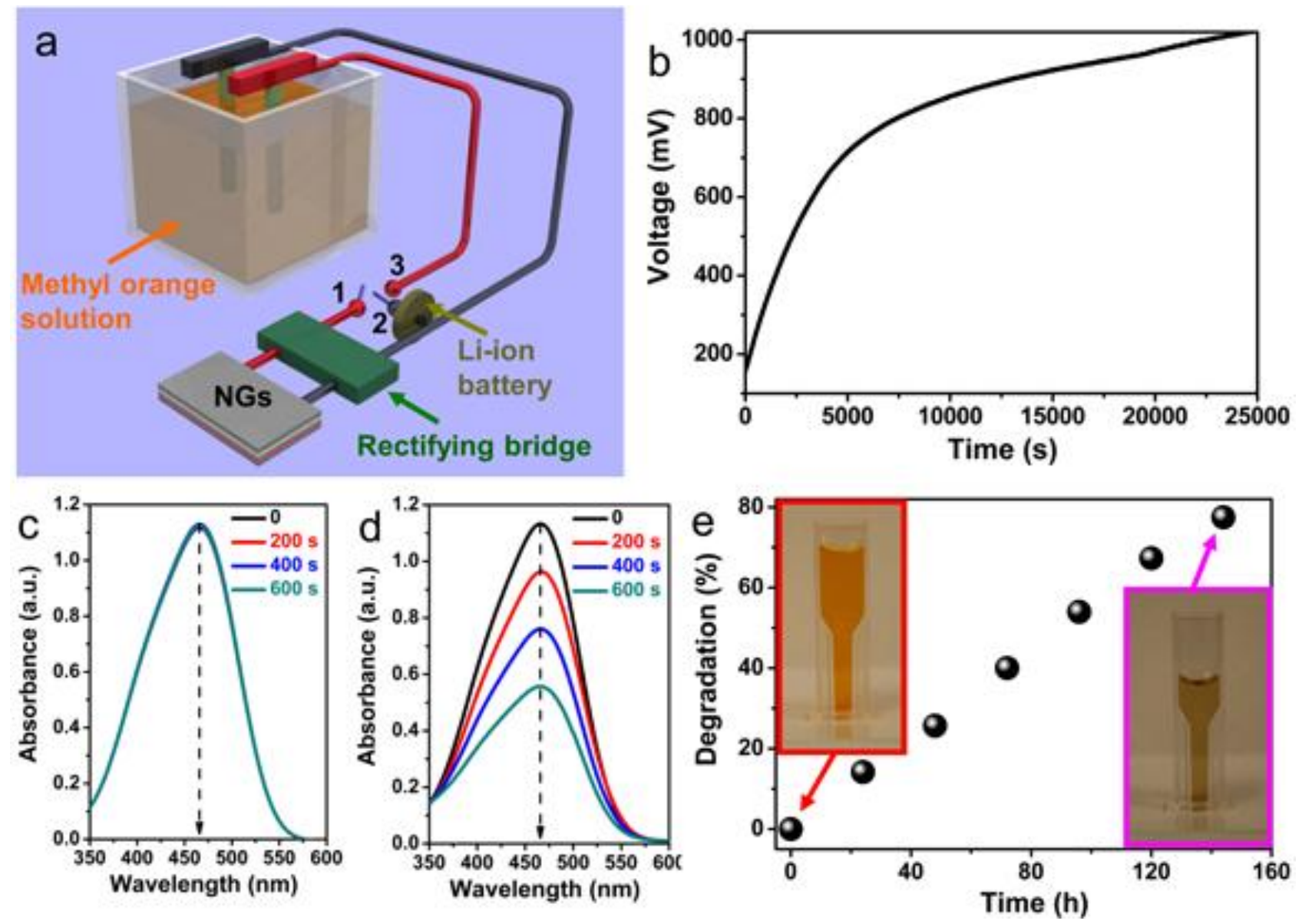

Figure 9. (a) Schematic diagram of the self-powered electro-degradation of methyl orange. (b) Charging curve of a Li-ion battery by using the hybrid energy cell. (c, d) Absorption spectra of the methyl orange solution without (c) and with (d) a Li-ion battery under the same time intervals. (e) Plot of degradation percentage versus the electro-degradation time. The inset shows the optical images of the methyl orange solution before and after the electro-degradation. Reproduced form ref 6. Copyright 2013 American Chemical Society. 

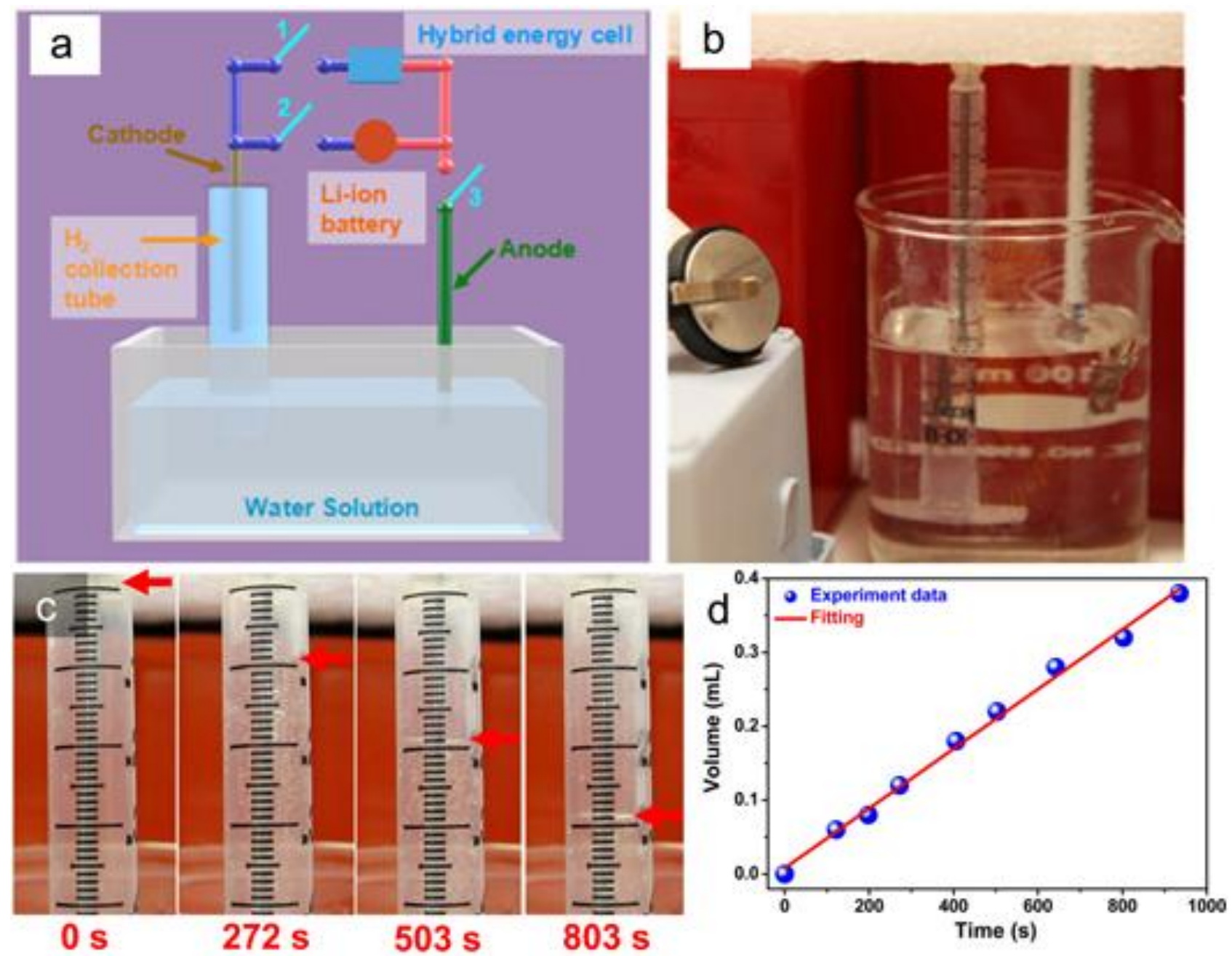

Figure 10. (a) Schematic diagram of the system for self-powered splitting of water to generate $\mathrm{H}_{2}$. (b) Optical image of the system for splitting of water into $\mathrm{H}_{2}$. (c) Optical images of the $\mathrm{H}_{2}$ collection tube under the different working times. (d) Corresponding volume of the produced $\mathrm{H}_{2}$ under different working times. Reproduced from ref. 9. Copyright 2013 Royal Society of Chemistry. 

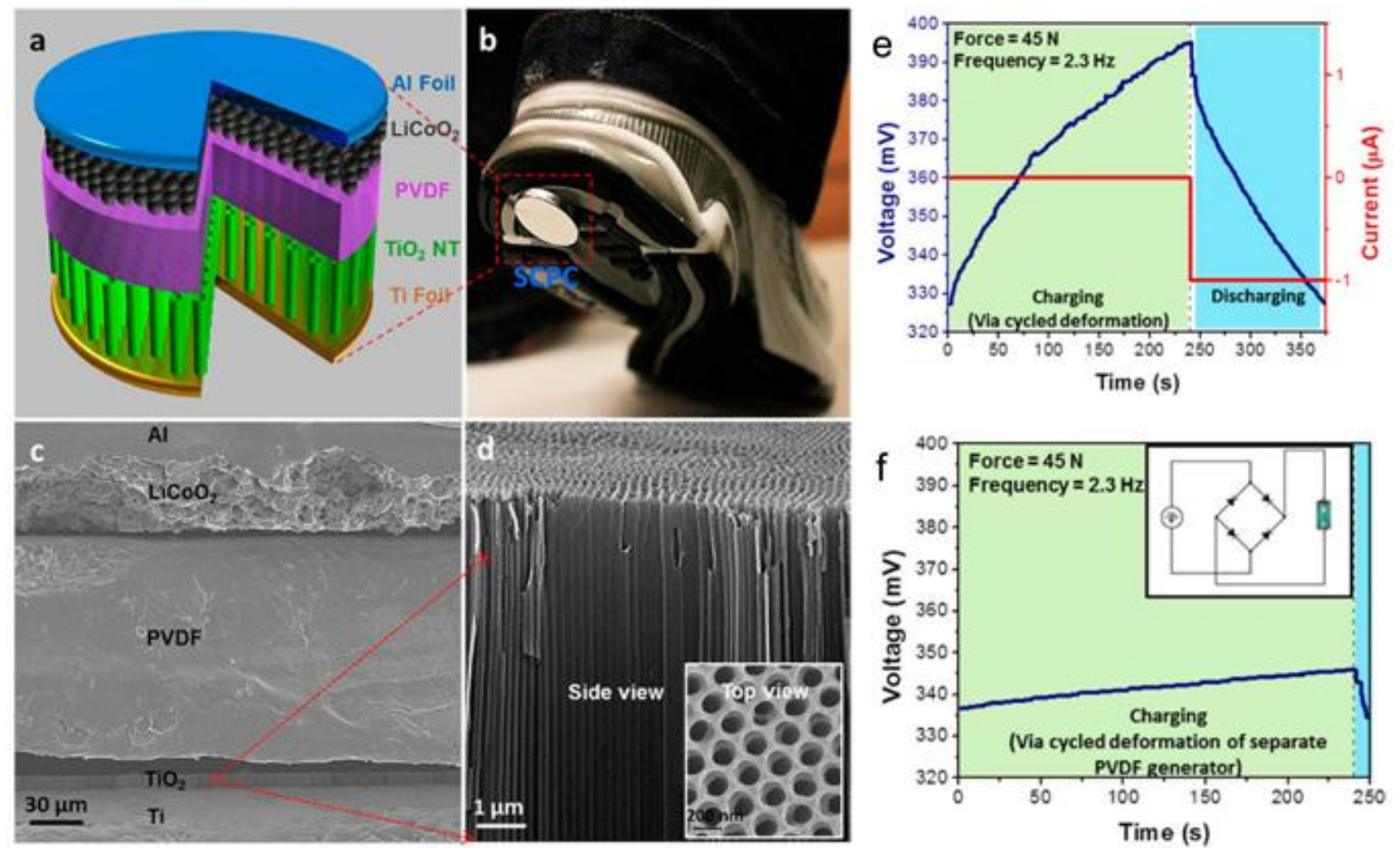

Figure 11. (a) Schematic diagram showing the design and structure of the self-charging power cell. (b) Sticking a power cell on the bottom of a shoe, the compressive energy generated by walking can be converted and stored directly by device. (c) Cross-sectional SEM image of the self-charging power cell. (d) Enlarged view of the aligned $\mathrm{TiO}_{2}$ nanotubes. (e) A typical self-charging process simply by applying cycled mechanical compressive strain to the device. (f) The charging curve by using an external PVDF generator for 4 min. Reproduced from ref. 15. Copyright 2012 American Chemical Society. 


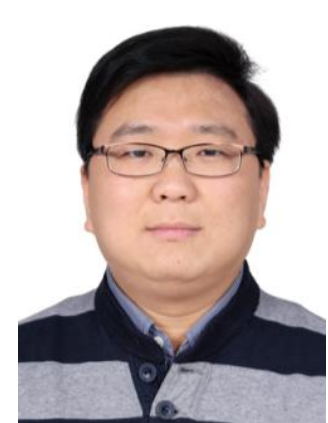

Ya Yang received his Ph.D. in Materials Science and Engineering from University of Science and Technology Beijing, China. He is currently a research scientist at Beijing Institute of Nanoenergy and Nanosystems, CAS. His main research interests focus on the field of pyroelectric nanogenerators, triboelectric nanogenerators, thermoelectric nanogenerators, and solar cells for energy conversion, storage and some novel applications.

Details can be found at: http://sourcedb.binn.cas.cn/zw/zjrck/yjy/201308/t20130814_3911917.html

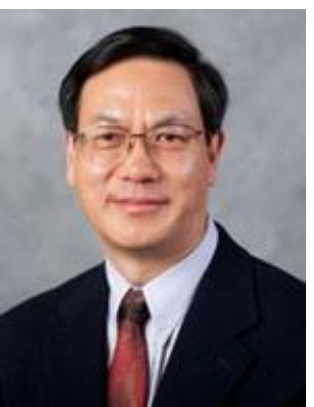

Zhong Lin (ZL) Wang received his Ph.D. from Arizona State University in physics. He now is the Hightower Chair in Materials Science and Engineering, Regents' Professor, Engineering Distinguished Professor and Director, Center for Nanostructure Characterization, at Georgia Tech. Dr. Wang has made original and innovative contributions to the synthesis, discovery, characterization and understanding fundamental physical properties of oxide nanobelts and nanowires, as well as applications of nanowires in energy sciences, electronics, optoelectronics and biological science. His discovery and breakthroughs in developing nanogenerators established the principle and technological roadmap for harvesting mechanical energy from the environment and biological systems for powering a personal electronics. His research on self-powered nanosystems has inspired the worldwide effort in academia and industry for studying energy of or micro- nano-systems, which is now a distinct disciplinary in energy research and future sensor networks. He coined and pioneered the field of piezotronics and piezo-phototronics by introducing piezoelectric potential gated charge transport process in fabricating new electronic and optoelectronic devices. Details can be found at: http://www.nanoscience.gatech.edu. 


\section{TOC}

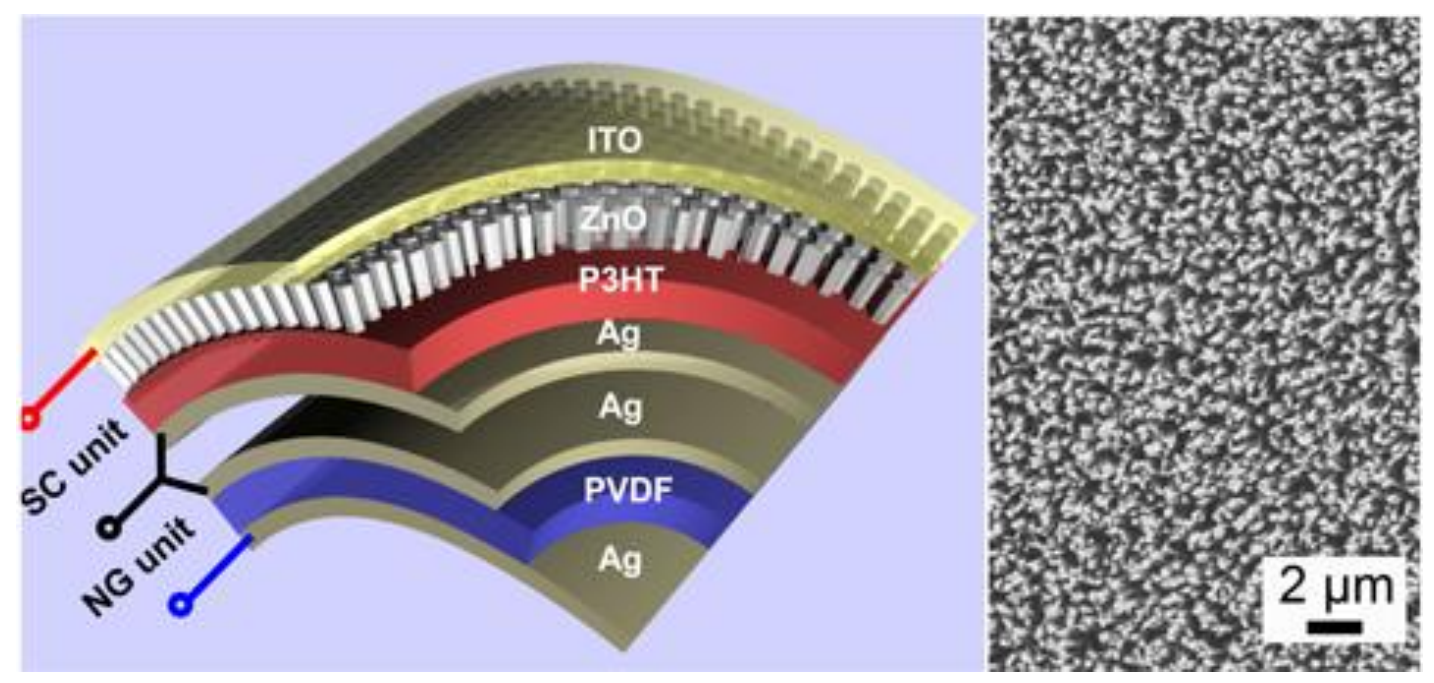

Harvesting multi-mode energies from our living environment is an effective approach for solving the power source issue of the sensors and some personal electronics. Usually, the mechanical, thermal, and solar energies can be extensively found in our living environment. However, these energies are not always available at the same time, which is depending on the weather, working conditions, and some other cases. The concept of a hybrid energy cell is to develop a technology to individually or simultaneously scavenge multi-mode energies from environment, so that the sensors or other devices can sustainably work without the external power sources. In this paper, we review the investigations about the hybrid energy cells that include the energy harvesting units such as the piezoelectric nanogenerator, triboelectric nanogenerator, pyroelectric nanogenerator, thermoelectric generator and solar cells. The fabricated hybrid energy cells have been utilized to light up some electronics and for some self-powered electro-chemical applications. The obtained energies can be also stored in Li-ion battery, where the hybrid structure between the nanogenerator and the Li-ion battery exhibits the better charging performance than that of the conventional charging method. These investigations are of critical importance for sensing, medical science, environmental monitoring, defense technology, and even personal electronics. 